\title{
Generalised Perspective Shape from Shading with Oren-Nayar Reflectance
}

Yong Chul Ju'

ju@tu-cottbus.de

Silvia Tozza²

tozza@mat.uniroma1.it

Michael Breuß ${ }^{1}$

breuss@tu-cottbus.de

Andrés Bruhn ${ }^{3}$

bruhn@vis.uni-stuttgart.de

Andreas Kleefeld ${ }^{1}$

kleefeld@tu-cottbus.de
${ }^{1}$ BTU Cottbus, Germany

${ }^{2}$ Sapienza - University of Rome, Italy

${ }^{3}$ University of Stuttgart, Germany

\begin{abstract}
In spite of significant advances in Shape from Shading (SfS) over the last years, it is still a challenging task to design SfS approaches that are flexible enough to handle a wide range of input scenes. In this paper, we address this lack of flexibility by proposing a novel model that extends the range of possible applications. To this end, we consider the class of modern perspective SfS models formulated via partial differential equations (PDEs). By combining a recent spherical surface parametrisation with the advanced non-Lambertian Oren-Nayar reflectance model, we obtain a robust approach that allows to deal with an arbitrary position of the light source while being able to handle rough surfaces and thus more realistic objects at the same time. To our knowledge, the resulting model is currently the most advanced and most flexible approach in the literature on PDE-based perspective SfS. Apart from deriving our model, we also show how the corresponding set of sophisticated Hamilton-Jacobi equations can be efficiently solved by a specifically tailored fast marching scheme. Experiments with medical real-world data demonstrate that our model works in practice and that is offers the desired flexibility.
\end{abstract}

\section{Introduction}

Shape from Shading (SfS) is defined as inferring the shape of an object depicted in a single input image given only the light reflectance and illumination in the scene. It is a classic task in computer vision and was pioneered by Horn $[\mathbf{Q}, \mathbf{Q}]$ using partial differential equations (PDEs), see $[\because, \square]$ for reviews of the field. Since then, there has been enormous progress in the area of SfS. In particular, the appropriate modelling of light reflectance and scene illumination has played a key role in the design of successful SfS approaches.

With respect to the light reflectance, the classical orthographic model of Horn [ $\mathrm{\theta}]$ based on Lambertian surfaces was soon replaced by advanced non-Lambertian reflectance models 
that offer an improved capability to handle realistic scenarios; see e.g. [Q, 四]. In this context, the reflectance model of Oren-Nayar turned out to be particularly useful [ $[\square, \square, \square]$. It is a general model for diffuse reflectance and has been studied in detail in the frameworks concerning orthographic SfS [四]. Relying on perspective instead of orthographic projection, a different class of modern SfS approaches has emerged in the last years [D], most of them formulated again in terms of PDEs $[\square, \square, \square]$. By assuming a Lambertian surface and the illumination given by a point light source located at the optical centre of the camera, they feature many well-posedness properties in contrast to their orthographic counterparts. In particular, the quadratic light fall-off due to inverse square law turned out to be very useful in this context $[\boldsymbol{G}, \mathbb{Q}]$ ]. Recently, such perspective SfS models with the light source located at the optical centre of the camera have been extended to non-Lambertian reflectance models $[\square, \mathbb{Q}, \mathbf{\theta}]$ such as the aforementioned Oren-Nayar model $[\nabla]$. This model has also been investigated in [四] for use with the highly efficient fast marching (FM) method [四].

Turning to more general illumination scenarios, there have been recent works on perspective SfS with Lambertian reflectance for a point light source not located at the optical centre. While Wu et al. [因] presented a multi-image optimisation framework, Galliani et al. [వ] proposed a PDE-based approach for a single input image that leads to an efficient FM implementation. However, no advanced non-Lambertian reflectance models have been employed in the context of general illumination settings so far.

Summarising, in the previous work on perspective SfS, approaches for non-Lambertian surfaces as well as for point light sources not co-located with the projection centre have only been considered individually. This shows that it is an extremely challenging task to relax the original SfS setup. In particular, there have been no attempts so far to combine a non-Lambertian reflectance model with a general illumination setup - even though, from an application viewpoint, the framework should evidently be as flexible as possible.

In the current paper, we advance the field by successfully merging the aforementioned two paths of research. (i) As in the work of Galliani et al. [Q] we consider the use of a spherical coordinate system centred at the point light source. (ii) Instead of employing a simple Lambertian reflectance model, we formulate a new brightness equation based on the advanced reflectance model of Oren and Nayar. The resulting approach is a sophisticated PDE belonging to the class of Hamilton-Jacobi equations (HJEs) where the solution has to be understood in a viscosity sense, cf. $[\boldsymbol{\square}, \square, \mathbb{\square}, \mathbb{\square}, \mathbf{⿴}]$. Apart from creating a very general and flexible approach, setting up our model in this way offers a third advantage: (iii) since for (advanced) diffuse reflectance models the brightest points correspond approximately to local minima of the depth [四], it allows us to extend the efficient FM algorithm proposed in [D] to our model - although our HJEs are significantly more complex. Experiments with medical real-world input images from endoscopy allow us to verify the useful properties of our approach, see e.g. [四, 四] for a similar application of SfS.

Our paper is organised as follows. First, we briefly recall the SfS setting in spherical coordinates and the Oren-Nayar reflectance model that serves as basis for our approach. Then, we proceed with the derivation of our new model and comment on its algorithmic realisation. After a presentation of computational results, we finally conclude our work.

\section{Perspective SfS Based on Spherical Coordinates}

While most approaches in the SfS literature are still based on the assumption that the light source is located at the optical centre of the camera, we follow Galliani et al. [ $\square$ ] and consider 
a general setup in which the light source is allowed to be located anywhere in the scene. The corresponding geometry is depicted in Fig. 1. It is based on a spherical coordinate system whose origin has been positioned at the location of the light source.

The main idea is to represent the Cartesian vector $\mathbf{r}=\overrightarrow{L S}$, i.e. the distance from the light source to the surface, via two angles $\theta$ and $\varphi$, respectively, as well as a radius $r$ :

$$
\mathbf{r}=R_{x_{3}}(\theta) R_{x_{2}}(\varphi)\left[\begin{array}{l}
0 \\
0 \\
r
\end{array}\right]=\left[\begin{array}{ccc}
\cos \theta \cos \varphi & -\sin \theta & \cos \theta \sin \varphi \\
\sin \theta \cos \varphi & \cos \theta & \sin \theta \sin \varphi \\
-\sin \varphi & 0 & \cos \varphi
\end{array}\right]\left[\begin{array}{l}
0 \\
0 \\
r
\end{array}\right] .
$$

Here, the two matrices $R_{x_{3}}(\theta)$ and $R_{x_{2}}(\varphi)$ represent rotations about the $x_{3}$ - and $x_{2}$-axis, respectively. The corresponding orthonormal basis is then given by

$$
\mathbf{e}_{\varphi}=\left[\begin{array}{c}
\cos \varphi \cos \theta \\
\cos \varphi \sin \theta \\
-\sin \varphi
\end{array}\right], \quad \mathbf{e}_{\theta}=\left[\begin{array}{c}
-\sin \theta \\
\cos \theta \\
0
\end{array}\right], \quad \mathbf{e}_{r}=\left[\begin{array}{c}
\sin \varphi \cos \theta \\
\sin \varphi \sin \theta \\
\cos \varphi
\end{array}\right]
$$

where

$$
\varphi=\arccos \left(r_{3} / \sqrt{r_{1}^{2}+r_{2}^{2}+r_{3}^{2}}\right) \quad \text { and } \quad \theta=\arctan \left(r_{2} / r_{1}\right)
$$

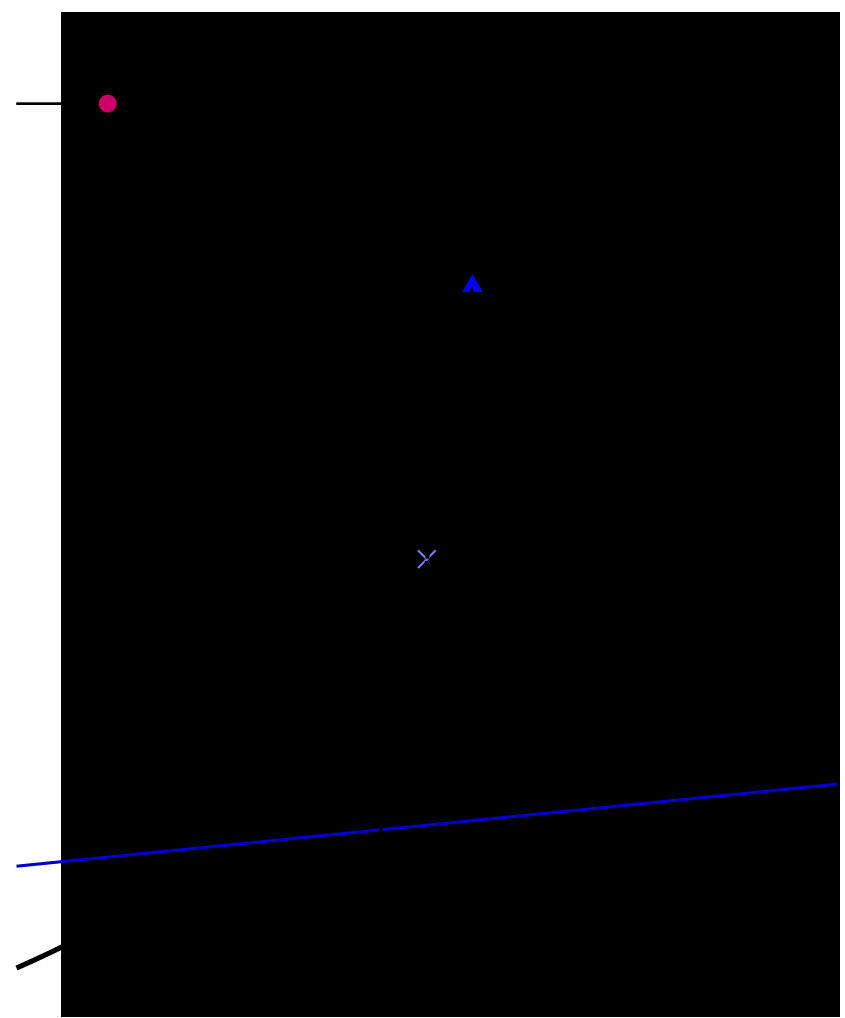

Figure 1: General SfS setup with arbitrary position of the light source. Adapted from [Q]. 
In this basis defined by spherical coordinates, the position vector from the light source to a surface point can be compactly written as:

$$
\left[\begin{array}{lll}
r_{1} & r_{2} & r_{3}
\end{array}\right]^{\top}=: \mathbf{r}:=r \mathbf{e}_{r} \quad \text { with } \quad r=\sqrt{r_{1}^{2}+r_{2}^{2}+r_{3}^{2}} .
$$

Having parametrised the unknown surface in spherical coordinates, we now have to compute the surface normal for each pixel of the input image. This can be done by first determining the vectors defining the tangent plane - these vectors are given by the derivatives of the surface with respect to the two directions orthogonal to $\mathbf{r}$, namely $\varphi$ and $\theta-$ and then by computing the cross product to obtain the corresponding normal vector. Using the definition of $\mathbf{r}$ from (4), the surface normal vector can be determined as

$$
\mathbf{n}=\frac{\partial\left(r \mathbf{e}_{r}\right)}{\partial \theta} \times \frac{\partial\left(r \mathbf{e}_{r}\right)}{\partial \varphi}=r \frac{\partial r}{\partial \theta}\left(\mathbf{e}_{r} \times \frac{\partial \mathbf{e}_{r}}{\partial \varphi}\right)+r \frac{\partial r}{\partial \varphi}\left(\frac{\partial \mathbf{e}_{r}}{\partial \theta} \times \mathbf{e}_{r}\right)+r^{2}\left(\frac{\partial \mathbf{e}_{r}}{\partial \theta} \times \frac{\partial \mathbf{e}_{r}}{\partial \varphi}\right) .
$$

By noting from (2) that $\frac{\partial \mathbf{e}_{r}}{\partial \varphi}=\mathbf{e}_{\varphi}$ and $\frac{\partial \mathbf{e}_{r}}{\partial \theta}=\sin \varphi \mathbf{e}_{\theta}$, since $\left(\mathbf{e}_{\varphi}, \mathbf{e}_{\theta}, \mathbf{e}_{r}\right)$ constitutes a righthanded coordinate system, we finally obtain

$$
\mathbf{n}=r \frac{\partial r}{\partial \theta} \mathbf{e}_{\theta}+r \sin \varphi \frac{\partial r}{\partial \varphi} \mathbf{e}_{\varphi}-r^{2} \sin \varphi \mathbf{e}_{r}
$$

This information is required later on in the reflectance model to establish the connection between the known image brightness and the unknown local orientation of the surface.

\section{Perspective Oren-Nayar SfS Brightness Equation}

After we have discussed a suitable parametrisation of the object surface as well as the surface normal, let us now explain the Oren-Nayar reflectance model. In contrast to the standard Lambertian case that assumes the object surface to be ideally diffuse, the advanced OrenNayar model [ $\square, \mathbb{\square}, \mathbb{\square}, \mathbb{\square}, \mathbb{0}]$ explicitly allows to handle general rough surfaces. The idea of this model is to represent a rough surface as an aggregation of V-shaped cavities, each with Lambertian reflectance properties. Assuming the slopes of these cavities to be Gaussian distributed, the roughness of a surface can be characterised by a single parameter, namely the standard deviation $\sigma$ of its slope distribution.

For determining the irradiance, we consider additionally the inverse square law for the light fall-off. Thus, we obtain the following brightness equation for the Oren-Nayar model [Q, ㅁ] ]:

$$
I(\mathbf{x})=\frac{1}{r^{2}} \cos \left(\theta_{i}\right)\left(A+B \sin (\alpha) \tan (\beta) \max \left[0, \cos \left(\varphi_{i}-\varphi_{r}\right)\right]\right),
$$

where the two non-negative terms

$$
A=1-0.5 \sigma^{2}\left(\sigma^{2}+0.33\right)^{-1} \text { and } B=0.45 \sigma^{2}\left(\sigma^{2}+0.09\right)^{-1}
$$

depend on the statistics of the cavities via the roughness parameter $\sigma$. In this context, $\theta_{i}$ represents the angle between the unit surface normal $\mathbf{N}$ and the light source direction $\mathbf{L}, \theta_{r}$ stands for the angle between the unit surface normal $\mathbf{N}$ and the camera direction $\mathbf{V}, \varphi_{i}$ is the angle between the projection of the light source direction $\mathbf{L}$ and the $x_{1}$ axis onto the $\left(x_{1}, x_{2}\right)$ plane, $\varphi_{\mathrm{r}}$ denotes the angle between the projection of the camera direction $\mathbf{V}$ and the $x_{1}$ axis onto the $\left(x_{1}, x_{2}\right)$-plane, and the two variables $\alpha=\max \left[\theta_{i}, \theta_{r}\right], \beta=\min \left[\theta_{i}, \theta_{r}\right]$ stand for the maximum and minimum of the angles $\theta_{i}$ and $\theta_{r}$, respectively. 


\section{Perspective Oren-Nayar SfS in Spherical Coordinates}

In order to derive the nonlinear HJEs that describe our new model, we have to formulate the brightness equation of the Oren-Nayar reflectance model in (7) using the parametrisation in terms of spherical coordinates that we derived in Section 2. As a first step towards this goal, we have to determine the direction $\mathbf{L}$ of the light source and the viewing direction $\mathbf{V}$ of the camera. Following Fig. 1, these two directions can be computed as

$$
\mathbf{L}=-\mathbf{e}_{r} \quad \text { and } \quad \mathbf{V}=\overrightarrow{S C}=\overrightarrow{L C}-\overrightarrow{L S}=\left(v_{1}-r\right) \mathbf{e}_{r}+v_{2} \mathbf{e}_{\varphi}+v_{3} \mathbf{e}_{\theta},
$$

where

$$
v_{1}=\sqrt{c_{1}^{2}+c_{2}^{2}+c_{3}^{2}}, \quad v_{2}=\arccos \left(-c_{3} / \sqrt{c_{1}^{2}+c_{2}^{2}+c_{3}^{2}}\right), \quad v_{3}=\arctan \left(c_{2} / c_{1}\right) .
$$

Knowing the surface normal $\mathbf{n}$ from Eq. (6) as well as the light direction $\mathbf{L}$ and the viewing direction $\mathbf{V}$, we can then reformulate all trigonometric expressions occurring in the OrenNayar brightness equation (7) in terms of spherical coordinates. Using the relation

$$
\nabla r:=\nabla_{(\theta, \varphi)} r=\frac{1}{r}\left(\frac{\partial r}{\partial \varphi}\right) \mathbf{e}_{\varphi}+\frac{1}{r \sin \varphi}\left(\frac{\partial r}{\partial \theta}\right) \mathbf{e}_{\theta},
$$

yields

$$
\begin{aligned}
& \cos \left(\theta_{i}\right)=\mathbf{N} \cdot \mathbf{L}=\frac{\mathbf{n}}{|\mathbf{n}|} \cdot \mathbf{L}=\left(|\nabla r|^{2}+1\right)^{-1 / 2}, \\
& \cos \left(\theta_{r}\right)=\mathbf{N} \cdot \mathbf{V}=\left(\frac{1}{r \sin \varphi} \frac{\partial r}{\partial \theta} v_{3}+\frac{1}{r} \frac{\partial r}{\partial \varphi} v_{2}+r-v_{1}\right)\left(|\nabla r|^{2}+1\right)^{-1 / 2},
\end{aligned}
$$

with $\sin \left(\theta_{i}\right)=\sqrt{1-\cos ^{2}\left(\theta_{i}\right)}$ and $\sin \left(\theta_{r}\right)=\sqrt{1-\cos ^{2}\left(\theta_{r}\right)}$ which, in particular, allows us to rewrite $\sin (\alpha)$ and $\tan (\beta)$. What still remains to be computed is the expression $\cos \left(\varphi_{i}-\varphi_{r}\right)$. To this end, we calculate the projections of the directions $\mathbf{L}$ and $\mathbf{V}$ onto the $\left(x_{1}, x_{2}\right)$-plane. This is realised by setting $\varphi=\frac{\pi}{2}$ and, consequently, by reducing $v_{1}$ and $r$ defined in (10) and (4) to their first two components. Defining these projections of $\mathbf{L}$ and $\mathbf{V}$ as $\hat{\mathbf{I}}$ and $\hat{\mathbf{v}}$, respectively, we obtain

$$
\cos \left(\varphi_{i}-\varphi_{r}\right)=\hat{\mathbf{l}} \cdot \hat{\mathbf{v}}=\sqrt{r_{1}^{2}+r_{2}^{2}}-\sqrt{c_{1}^{2}+c_{2}^{2}} .
$$

Finally, we are in the position to rewrite Eq. (7) entirely in spherical coordinates. However, since there are several min and $\max$ operators involved, we have to distinguish four different cases. These cases are given by Eqs. (15)-(18). For each case we have listed the corresponding implications, the resulting brightness equation as well as the HJE that we finally have to solve. While Case 4 resembles the single Lambertian PDE in [ $\square$ ], the HJEs for the other cases are significantly more complex. This makes explicit that our general SfS approach with Oren-Nayar reflectance model is substantially more challenging than previous approaches based on the Lambertian assumption. Moreover, please note that Case 3 is actually a special case of Case 1 . However, since this case exactly describes the standard setting, where the light source is located at the optical centre of the camera, we decided to list it separately. Finally, we would like to mention that the angle between the light direction $\mathbf{L}$ and the viewing direction $\mathbf{V}$ cannot be larger or equal to $\pi$. This, however, is evident, since otherwise we cannot project these vectors onto the $\left(x_{1}, x_{2}\right)$-plane. 
Case 1: $\theta_{i} \geq \theta_{r}$ and $\left(\varphi_{i}-\varphi_{r}\right) \in\left[0, \frac{\pi}{2}\right) \cup\left(\frac{3}{2} \pi, 2 \pi\right]$

1. Implication: $\max \left[0, \cos \left(\varphi_{i}-\varphi_{r}\right)\right]=\cos \left(\varphi_{i}-\varphi_{r}\right)$

2. Brightness equation: $I(\mathbf{x})=\frac{1}{r^{2}} \cos \left(\theta_{i}\right)\left(A+B \cos \left(\varphi_{i}-\varphi_{r}\right) \sin \left(\theta_{i}\right) \frac{\sin \left(\theta_{r}\right)}{\cos \left(\theta_{r}\right)}\right)$

3. Hamilton-Jacobi equation:

$$
r^{2} I-\frac{A}{\sqrt{|\nabla r|^{2}+1}}-\frac{B(\hat{\mathbf{l}} \cdot \hat{\mathbf{v}})|\nabla r|}{|\nabla r|^{2}+1} \frac{\sqrt{|\nabla r|^{2}+1-\left(\frac{1}{r \sin \varphi} \frac{\partial r}{\partial \theta} v_{3}+\frac{1}{r} \frac{\partial r}{\partial \varphi} v_{2}+r-v_{1}\right)^{2}}}{\left(\frac{1}{r \sin \varphi} \frac{\partial r}{\partial \theta} v_{3}+\frac{1}{r} \frac{\partial r}{\partial \varphi} v_{2}+r-v_{1}\right)}=0
$$

Case 2: $\theta_{i}<\theta_{r}$ and $\left(\varphi_{i}-\varphi_{r}\right) \in\left[0, \frac{\pi}{2}\right) \cup\left(\frac{3}{2} \pi, 2 \pi\right]$

1. Implication: $\max \left[0, \cos \left(\varphi_{i}-\varphi_{r}\right)\right]=\cos \left(\varphi_{i}-\varphi_{r}\right)$

2. Brightness equation: $I(\mathbf{x})=\frac{1}{r^{2}} \cos \left(\theta_{i}\right)\left(A+B \cos \left(\varphi_{i}-\varphi_{r}\right) \sin \left(\theta_{r}\right) \frac{\sin \left(\theta_{i}\right)}{\cos \left(\theta_{i}\right)}\right)$

3. Hamilton-Jacobi equation:

$$
r^{2} I-\frac{A}{\sqrt{|\nabla r|^{2}+1}}-\frac{B(\hat{\mathbf{l}} \cdot \hat{\mathbf{v}})|\nabla r|}{|\nabla r|^{2}+1} \sqrt{|\nabla r|^{2}+1-\left(\frac{1}{r \sin \varphi} \frac{\partial r}{\partial \theta} v_{3}+\frac{1}{r} \frac{\partial r}{\partial \varphi} v_{2}+r-v_{1}\right)^{2}}=0
$$

Case 3: $\theta_{i}=\theta_{r}$ and $\varphi_{i}=\varphi_{r}$

1. Implications: $\quad \theta:=\theta_{i}=\theta_{r}=\alpha=\beta$ and $\max \left[0, \cos \left(\varphi_{i}-\varphi_{r}\right)\right]=1$

2. Brightness equation: $I(\mathbf{x})=\frac{1}{r^{2}} \cos (\theta)\left(A+B \frac{\sin \left(\theta^{2}\right)}{\cos (\theta)}\right)$

3. Hamilton-Jacobi equation: $\quad r^{2} I\left(|\nabla r|^{2}+1\right)-A \sqrt{|\nabla r|^{2}+1}-B|\nabla r|^{2}=0$

Case 4: For any $\theta_{i}, \theta_{r}$, and $\left(\varphi_{i}-\varphi_{r}\right) \in\left[\frac{\pi}{2}, \frac{3}{2} \pi\right]$

1. Implication: $\max \left[0, \cos \left(\varphi_{i}-\varphi_{r}\right)\right]=0$

2. Brightness equation: $I(\mathbf{x})=\frac{1}{r^{2}} A \cos \left(\theta_{i}\right)$

3. Hamilton-Jacobi equation: $\quad I \sqrt{|\nabla r|^{2}+1}-\frac{A}{r^{2}}=0$ 


\section{Fast Marching Solver}

After we have derived the brightness equation for the Oren-Nayar reflection model with general position of the light source, let us now discuss how we can solve the resulting set of HJEs for the unknown radial distance $r$. In order to allow for an efficient computation, we rely on a variant of the fast marching (FM) schemes which are among the fastest solvers for PDEs available in the literature $[\square]$. Such schemes start from critical points, i.e. points that are local minima of the depth, and then propagate the solution to the remaining points on the surface. Thereby, at each grid point, the corresponding nonlinear PDE from Eq. (15)-(18) has to be solved iteratively for the unknown radial distance. Since our HJEs are formulated in a spherical coordinate system that is usually not centred at the camera position, the location where the radiance data $I$ has to be evaluated depends on $r$. This, however, prevents the application of standard FM schemes, since they assume $I$ not to change during the iterations.

Recently, Galliani et al. [0] proposed a specifically adapted variant of FM for spherical coordinates. Although their SfS model is restricted to the Lambertian case and the corresponding single HJE is significantly simpler than our set of HJEs, we can still make use of the basic strategy of their approach:

(i) In a first step, we identify critical points on the surface based on their brightness values. Since such points denote local minima with respect to their distance to the light source, we know that $\nabla r=0$. This allows us to solve the set of HJEs at those locations locally for the radial depth $r$, i.e. without considering information from neighbouring pixels. Solving for $r$ can either be done iteratively (Case 1 and 2) or directly (Case 3 and 4).

(ii) After computing the depth values at critical points, we propagate the information to other points in terms of $\theta$ and $\varphi$ by sequentially updating the depth values at neighbouring locations via solving the corresponding HJE there. As proposed in [ $\square]$, we apply the iterative update strategy from [ [Q] $]$ making use of the regula falsi method that has been originally employed in the context of nonlinear HJEs in Euclidean coordinates. This requires to discretise our HJEs for which we use a standard upwind scheme as described in [ $\mathrm{Z}$ ] $]$. Moreover, since $x_{1}$ and $x_{2}$ depend on the radial depth $r$, we have to update the sample location and thus the value of $I\left(x_{1}(\theta, \varphi, r), x_{2}(\theta, \varphi, r)\right)$ at each iteration within our iterative framework. The sampling at subpixel locations is done using bilinear interpolation. The iterations are stopped if the residual of the equation drops below a certain threshold $T$. In our case, we use $T=10^{-3}$.

\section{Experimental Evaluation}

In order to evaluate the quality and robustness of our model, we have used endoscopic images provided in [Ш]. Since model parameters in SfS are in general unknown, experiments with such real-world data are highly challenging; see e.g. [四, 四]. In particular, the position of the camera relative to the position of the light source is typically not provided with such data sets, since standard SfS models are restricted to the assumption that both positions coincide (which is often not true by construction). However, in contrast to such techniques, our model can handle scenarios where the position of the light source is actually not located at the optical centre of the camera. Thus, for our experiments, we had to rely on a rough estimation of the correct position by visually inspecting the images. Hence, we assumed that the light source is located at the origin based on Fig. 1 and chose the position of the camera nearby but not too close to the origin. Since it was already shown in [0] that a model with flexible position of the light source can give significant advantages over a standard SfS approach, we 
focus in the following on the other two important aspects of our novel method: the visual quality of the reconstruction as well as its robustness with respect to parameter variations.

In our first experiment, we evaluated the impact of the spherical resolution on the quality of the reconstruction. To this end, we used uniform grids in the $\varphi$ - $\theta$-domain with mesh widths $\delta_{\varphi \theta}=\Delta \varphi=\Delta \theta$ and computed the results for different resolutions. As we can see from Fig. 2, the reconstruction quality improves significantly when increasing the resolution, i.e. when refining the grid. However, one should keep in mind that the actual quality is limited by the resolution of the input image which is repeatedly evaluated at sub-pixel positions during our FM computation. Moreover, one should note that, due to choice that the light source is at the origin of our coordinate system, the reconstruction is computed from the viewpoint of the light source. This explains the slight shift of the reconstruction with respect to the input image.

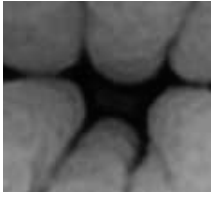

(a) Input image $(115 \times 106)$.

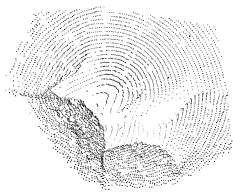

(b) $\delta_{\varphi \theta}=0.025$.

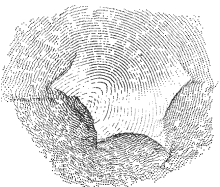

(c) $\delta_{\varphi \theta}=0.0175$.

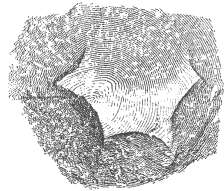

(d) $\delta_{\varphi \theta}=0.0125$.

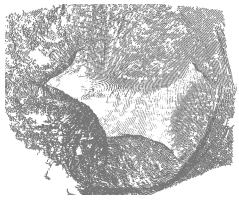

(e) $\delta_{\varphi \theta}=0.0075$.

Figure 2: Reconstruction of gastric antrum with $\sigma=\frac{\pi}{6}$.

In a second experiment, we investigated the robustness of the reconstruction with respect to the choice of the roughness parameter $\sigma$ for the Oren-Nayar reflectance model. To this end, we have used four different input images and computed the reconstructions for different values of $\sigma$ accordingly. The Figs. 3-6 show the reconstruction of the duodenum, the esophagus, the papilla of Vater, and the stomach of lining, respectively. As we can see, the model gives very reasonable results and behaves in a well-posed manner, i.e. the results are stable and depend continuously on $\sigma$. This is very important for performing SfS computations with real-world input images, since the correct value of $\sigma$ is typically not known.

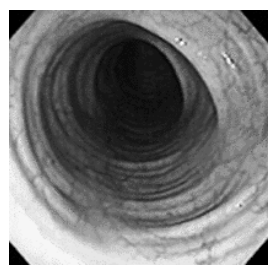

(a) Input image $(211 \times 208)$.

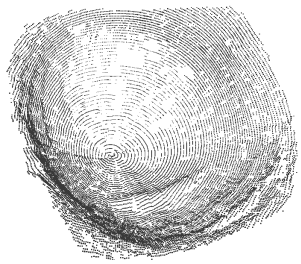

(b) $\sigma=\frac{\pi}{6}$.

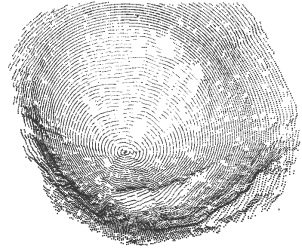

(c) $\sigma=\frac{\pi}{4}$.

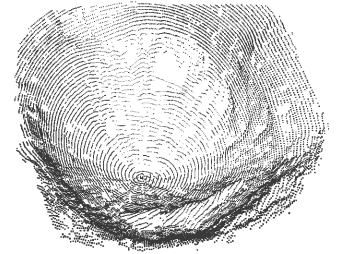

(d) $\sigma=\frac{\pi}{2}$.

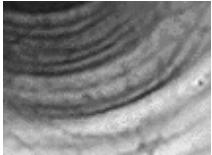

(e) Input image (cropped, $106 \times 78$ )

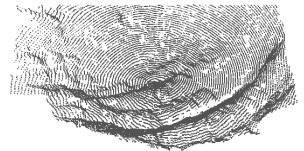

(f) $\sigma=\frac{\pi}{6}$.

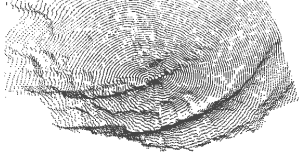

(g) $\sigma=\frac{\pi}{4}$.

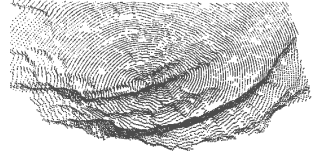

(h) $\sigma=\frac{\pi}{2}$.

Figure 3: Reconstruction of the duodenum $\delta_{\varphi \theta}=0.0125$, grid size $504 \times 504$. 


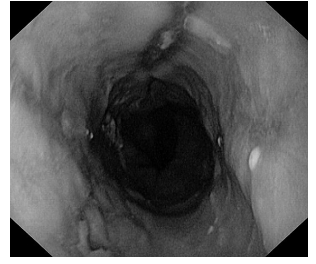

(a) Input image $(586 \times 502)$.

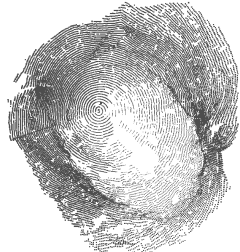

(b) $\sigma=\frac{\pi}{6}$.

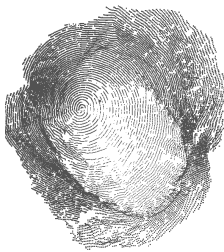

(c) $\sigma=\frac{\pi}{4}$.

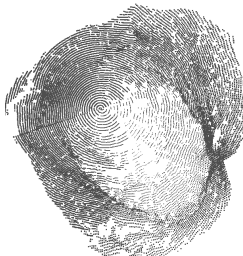

(d) $\sigma=\frac{\pi}{2}$.

Figure 4: Reconstruction of the esophagus $\delta_{\varphi \theta}=0.0125$, grid size $504 \times 504$.

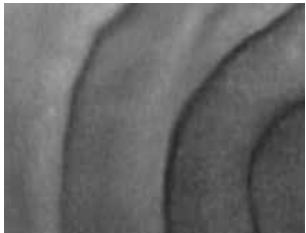

(a) Input image $(210 \times 160)$.

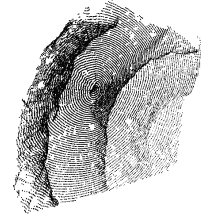

(b) $\sigma=\frac{\pi}{6}$.

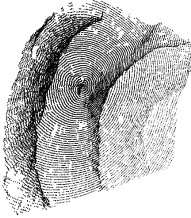

(c) $\sigma=\frac{\pi}{4}$

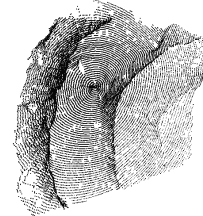

(d) $\sigma=\frac{\pi}{2}$.

Figure 5: Reconstruction of the papilla of Vater $\delta_{\varphi \theta}=0.0125$, grid size $504 \times 504$.

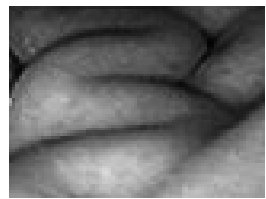

(a) Input image $(80 \times 71)$.

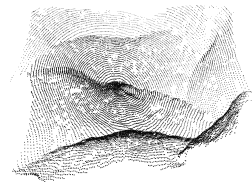

(b) $\sigma=\frac{\pi}{6}$.

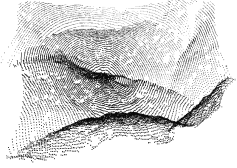

(c) $\sigma=\frac{\pi}{4}$

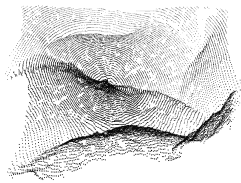

(d) $\sigma=\frac{\pi}{2}$.

Figure 6: Reconstruction of the stomach lining $\delta_{\varphi \theta}=0.0125$, grid size $504 \times 504$.

\section{Conclusion}

We have presented a perspective SfS model that combines the advantage of freely selecting the position of the light source with the robustness of an advanced non-Lambertian reflectance model. By considering both a general light source setup and an improved reflectance model, the resulting approach is one of the most advanced and flexible models in the field. This is also validated by our experiments with medical real-world images that gave good results. Despite of these results, we found that the relatively large number of unknown parameters still makes conducting experiments difficult. Besides camera, reflectance and lighting parameters that have to be roughly estimated, the spherical resolution of the result and the iteration and threshold parameters may need some manual adjustment. This is the price one has to pay for taking into account an advanced reflection model that leads to a set of relatively complex PDEs. Nevertheless, we believe that the combination of advanced models for light source setup and reflectance is definitely worthwhile. However, the reflectance model should not be more complicated than the one of Oren and Nayar. Otherwise a carefully controlled experimental environment is needed to achieve meaningful results; see [四].

Acknowledgements. This work has been partly funded by the Deutsche Forschungsgemeinschaft as a joint research project (BR 2245/3-1, BR 4372/1-1). 


\section{References}

[1] Gastrolab. http://www. gastrolab. net.

[2] A.H. Ahmed and A.A. Farag. A new formulation for shape from shading for nonLambertian surfaces. In Proc. IEEE Conference on Computer Vision and Pattern Recognition (CVPR), pages 1817-1824, 2006.

[3] S. Bakshi and Y.-H. Yang. Shape from shading for non-Lambertian surfaces. In Proc. IEEE International Conference on Image Processing (ICIP), pages 130-134, 1994.

[4] M. Breuß, E. Cristiani, J.-D. Durou, M. Falcone, and O. Vogel. Perspective shape from shading: Ambiguity analysis and numerical approximations. SIAM Journal on Imaging Sciences, 5(1):311-342, 2012.

[5] M.G. Crandall and P.-L. Lions. Viscosity solutions of Hamilton-Jacobi equations. Transactions of the American Mathematical Society, 277(1):1-42, 1983.

[6] J.-D. Durou, M. Falcone, and M. Sagona. Numerical methods for shape-from-shading: A new survey with benchmarks. Computer Vision and Image Understanding, 109(1): 22-43, 2008.

[7] S. Galliani, Y.-C. Ju, M. Breuß, and A. Bruhn. Generalised perspective shape from shading in spherical coordinates. In Proc. Scale Space and Variational Methods in Computer Vision (SSVM), pages 222-233, 2013.

[8] B.K.P. Horn. Shape From Shading: A Method for Obtaining the Shape of a Smooth Opaque Object From One View. PhD thesis, Massachusetts Institute of Technology, 1970.

[9] B.K.P. Horn and M.J. Brooks. Shape from Shading. The MIT Press, 1989.

[10] Y.-C. Ju, M. Breuß, A. Bruhn, and S. Galliani. Shape from shading for rough surfaces: Analysis of the Oren-Nayar model. In Proc. British Machine Vision Conference (BMVC), pages 104.1-104.11, 2012.

[11] R. Kimmel, K. Siddiqi, B. B. Kimia, and A. M. Bruckstein. Shape from shading: Level set propagation and viscosity solutions. International Journal of Computer Vision, 16: 107-133, 1995.

[12] T. Okatani and K. Deguchi. Shape reconstruction from an endoscope image by shape from shading technique for a point light source at the projection center. Computer Vision and Image Understanding, 66(2):119-131, 1997.

[13] M. Oren and S.K. Nayar. Diffuse reflectance from rough surfaces. In Proc. IEEE Conference on Computer Vision and Pattern Recognition (CVPR), pages 763-764, 1993.

[14] M. Oren and S.K. Nayar. Generalization of Lambert's reflectance model. In Proc. International Conference and Exhibition on Computer Graphics and Interactive Techniques (SIGGRAPH), pages 239-246, 1994.

[15] M. Oren and S.K. Nayar. Seeing beyond Lambert's law. In Proc. European Conference on Computer Vision (ECCV), pages 269-280, 1994. 
[16] M. Oren and S.K. Nayar. Generalization of the Lambertian model and implications for machine vision. International Journal of Computer Vision, 14(3):227-251, 1995.

[17] E. Prados and O.D. Faugeras. Perspective shape from shading and viscosity solutions. In Proc. IEEE International Conference on Computer Vision (ICCV), pages 826-831, 2003.

[18] E. Prados and O.D. Faugeras. Shape from shading: a well-posed problem? In Proc. IEEE Conference on Computer Vision and Pattern Recognition (CVPR), pages 870$877,2005$.

[19] H. Ragheb and E.R. Hancock. Surface radiance correction for shape from shading. Pattern Recognition, 38(10):1574-1595, 2005.

[20] E. Rouy and A. Tourin. A viscosity solutions approach to shape-from-shading. SIAM Journal on Numerical Analysis, 29(3):867-884, June 1992.

[21] J.A. Sethian. Level Set Methods and Fast Marching Methods. Cambridge University Press, 2nd edition, 1999.

[22] A. Tankus, N. Sochen, and Y. Yeshurun. Perspective shape-from-shading by fast marching. In Proc. IEEE Conference on Computer Vision and Pattern Recognition (CVPR), pages 43-49, 2004.

[23] A. Tankus, N. Sochen, and Y. Yeshurun. Shape-from-shading under perspective projection. International Journal of Computer Vision, 63(1):21-43, 2005.

[24] O. Vogel, M. Breuß, and J. Weickert. Perspective shape from shading with nonLambertian reflectance. In Proc. DAGM Symposium on Pattern Recognition, pages 517-526, 2008.

[25] G.-H. Wang, J.-Q. Han, and X.-M. Zhang. Three-dimensional reconstruction of endoscope images by a fast shape from shading method. Measurement Science and Technology, 20(12):125801, 2009.

[26] C. Wu, S. Narasimhan, and B. Jaramaz. A multi-image shape-from-shading framework for near-lighting perspective endoscopes. International Journal of Computer Vision, 86:211-228, 2010.

[27] R. Zhang, P.-S. Tsai, J.E. Cryer, and M. Shah. Shape from shading: A survey. IEEE Transactions on Pattern Analysis and Machine Intelligence, 21(8):690-706, 1999. 